\title{
Leveraging Electronic Tablets for General Pediatric Care
}

\section{A pilot study}

\author{
V. Anand'; S. McKee²; T.M. Dugan²; S.M. Downs ${ }^{2,3}$ \\ ${ }^{1}$ Pediatric Institute, Cleveland Clinic, Cleveland, $\mathrm{OH} ;{ }^{2}$ Childrens Health Services Research, School of Medicine, Indiana University, \\ IN; ${ }^{3}$ Regenstrief Institute for Healthcare, IN
}

\author{
Keywords \\ Electronic tablets, clinical decision support system, CDSS, pediatrics, children, EMR
}

\section{Summary}

Background: We have previously shown that a scan-able paper based interface linked to a computerized clinical decision support system (CDSS) can effectively screen patients in pediatric waiting rooms and support the physician using evidence based care guidelines at the time of clinical encounter. However, the use of scan-able paper based interface has many inherent limitations including lacking real time communication with the CDSS and being prone to human and system errors. An electronic tablet based user interface can not only overcome these limitations, but may also support advanced functionality for clinical and research use. However, use of such devices for pediatric care is not well studied in clinical settings.

Objective: In this pilot study, we enhance our pediatric CDSS with an electronic tablet based user interface and evaluate it for usability as well as for changes in patient questionnaire completion rates.

Methods: Child Health Improvement through Computers Leveraging Electronic Tablets or CHICLET is an electronic tablet based user interface. It is developed to augment the existing scan-able paper interface to our CDSS. For the purposes of this study, we deployed CHICLET in one outpatient pediatric clinic. Usability factors for CHICLET were evaluated via caregiver and staff surveys.

Results: When compared to the scan-able paper based interface, we observed an $18 \%$ increase or $30 \%$ relative increase in question completion rates using CHICLET. This difference was statistically significant. Caregivers and staff survey results were positive for using CHICLET in clinical environment.

Conclusions: Electronic tablets are a viable interface for capturing patient self-report in pediatric waiting rooms. We further hypothesize that the use of electronic tablet based interfaces will drive advances in computerized clinical decision support and create opportunities for patient engagement.

\section{Correspondence to:}

Vibha Anand, PhD

Assistant Professor of Pediatrics,

Department \& Center for Pediatric Rheumatology and Immunology,

Department of Quantitaive Health Sciences, Lerner College of Medicine of Case Western Reserve University, Cleveland Clinic

9500 Euclid Ave JJN3-01Cleveland, Ohio 44195

$\mathrm{Ph}: 216-442-5565$

Fax: 216-444-8023

Email: anandv@ccf.org; vanand@iupui.edu
Appl Clin Inform 2015; 6: 1-15

http://dx.doi.org/10.4338/ACl-2014-09-RA-0071

received: September 26, 2014

accepted: December 11, 2014

published: January 7, 2015

Citation: Anand V, McKee S, Dugan TM, Downs SM. Leveraging electronic tablets for general pediatric care - a pilot study. Appl Clin Inf 2015; 6: 1-15

http://dx.doi.org/10.4338/ACI-2014-09-RA-0071 


\section{Background}

With the growth of Health Information Technology (Health IT) and with increased adoption of Electronic Medical Records (EMR) [1], there has come the daunting task of designing interoperable and accessible eHealth applications. These applications are aimed at providing physicians with the information necessary to guide efficient care and to promote health across the life span [2]. This is especially true in pediatric settings; pediatricians are ideally positioned to participate in collaborative care in partnership with families and authoritative guidelines exist to assist them in their work. Furthermore, pediatricians can aptly translate scientific advances from diverse fields into innovative new models of disease prevention, health promotion and developmental enhancement [3]. However, within the context of an office visit, pediatricians often lag to deliver recommended services. This may be due to the tight time constraints of a typical office visit and a plethora of available guidelines. As a result, studies have shown that children receive fewer than $50 \%$ of recommended services in a typical office visit [4]. Computerized clinical decision support systems (CDSS) can help address this problem by comparing characteristics of individual patients to computerized knowledge bases of guidelines for purposes of generating patient-specific assessments and recommendations for physicians [5].

Over the last nine years, pediatricians and informatics professionals in our research group have developed and deployed a highly innovative CDSS - the Child Health Improvement through Computer Automation system (CHICA). CHICA is used in five busy pediatric practices at Indiana University Hospitals [6]. CHICA generates age-appropriate and patient specific screening questionnaires that patients or their caregivers complete in the waiting room. CHICA then combines patient's screening results with data from EMR to generate patient-specific recommendations and reminders for the physician at the time of the clinical encounter. In 2004, when CHICA was initially deployed, it was deployed with a scan-able paper based interface to generate patient specific documents we call Adaptive Turnaround Documents (ATAD) [7]. However, ATADs have some inherent limitations due to paper as a medium and paper scanning functionality. Although paper is highly intuitive $[8,9]$, relatively cheap and scalable, it is prone to both human and system errors [10]. Furthermore, it does not support real-time interaction with the computer providing clinical decision support, rather it must be scanned, and a new sheet of paper printed, with each interaction. This prevents the CDSS from "drilling down" for example, to ask more detailed questions of patient families for purposes of screening or knowing about their preferences or barriers to treatment options. Use of paper also allows for unstructured input, which may serve to inform the clinical encounter but cannot be easily interpreted by the computer for further decision support. Moreover, stray marks, damaged paper, misaligned scans, limited capacity of printer trays, and human labor are all additional liabilities of data capture by paper scanning. Despite these liabilities, paper was the only viable medium originally because the alternative was a desktop or laptop computer that would not fit into a high volume workflow of busy pediatric practices or be capable of seamlessly delivering decision support to the clinician's hands in the encounter. The advent of small electronic tablets has introduced a new option. Electronic tablets are small and intuitive like paper. In fact, they can emulate the look and feel of paper reasonably closely. Moreover, an electronic tablet can interact in realtime not only with its users but also with the computer driving the clinical decision support, allowing the computer to ask more detailed questions for screening. Therefore, using electronic tablets, it is now feasible to develop user interfaces that have most of the advantages of a paper but not its drawbacks.

\section{Objectives}

We had a unique opportunity to implement and test the hypothesis that by extending the current CHICA system to include an electronic tablet based interface - The Child Health Improvement through Computers Leveraging Electronic Tablets (CHICLET), the screening data completion rates would improve while satisfying user expectations. To the best of our knowledge, the feasibility of using electronic tablet devices to capture structured data from patients or their caregivers in pediatric waiting rooms, and to drive clinical decision support based on patient reported data at the point 
of care, has not been studied before. For this pilot study, we replaced one existing ATAD (form) in the existing CHICA system with a CHICLET based form for use in one pediatric practice of our healthcare system. We hypothesized that CHICLET would be a desirable user interface for pediatric care and that CHICLET could be effective in collecting patient reported data in pediatric waiting rooms. Furthermore, we hypothesized that use of electronic tablets over paper will improve questionnaire completion rates without interfering with clinic workflow.

\section{Methods}

\section{The CHICA System}

CHICA is a computer based decision support system for pediatric preventative care and disease management [11-15]. CHICA uses an open source electronic medical record platform [16] (www. openmrs.org) and serves as a front end to our EMR, the Regenstrief Medical Record System [17]. However, with a modest integration effort, CHICA also has the capability to work as a standalone application or together with another EMR. CHICA has been in use in four (now five) primary care pediatric practices and community health centers in our hospital system over the past decade. The system consists of

1. a knowledge base of guideline rules,

2. a repository of patient data,

3. a tailored document printing and scanning engine, and

4. business rules to direct communication as well as the printing and scanning of patient specific documents [11].

At each visit, CHICA generates two ATADs. An ATAD is a form for structured input which can be completed using hand-written marks. It is quite accurately read and interpreted using optical character recognition or OCR (www.verity.com) technology; however, each ATAD requires form development and customization in the OCR software. The first ATAD is the pre-screener form (PSF) which collects information from the patient/caregiver and from the nurse, before the physician encounter. PSF is printed in English on one side and Spanish on the other [18] and is generated for screening based on data from patient's electronic medical record and their age at visit. ( $>$ Figure 1) The second ATAD is the physician worksheet which provides reminders and collects data from the physician. To determine what information needs to be printed on each ATAD, CHICA employs a library of care guidelines encoded as Arden Syntax Medical Logic Module or MLMs [19, 20] - to evaluate logic against patient databases. Since time constraints limit the number of topics that can be addressed feasibly in a given patient encounter, CHICA employs a global prioritization scheme which limits the printed content to what is most relevant and important based on the expected value of information [21]. Additionally, CHICA generates Just-in-Time ATADs. These are generated for informational purposes (e.g. How to quit - smoking cessation handout) and as age appropriate screening instruments (e.g., the Ages and Stages Questionnaire [22], the Vanderbilt for Attention Deficit Hyperactivity Disorder Screeners [23], and the Modified Checklist for Autism in Toddlers or M-CHAT [24]). At the end of each visit, CHICA exports data from patient ATADs to the EMR for use in future care. Using ATADs as an interface, CHICA has been remarkably successful in meeting both patient and physician needs [25-30]. This success has been largely attributed to automating the process of screening in pediatric waiting rooms and alerting the physicians to the screened risk while decreasing the burden of identifying relevant guidelines for physicians.

\section{Child Health Improvement through Computers Leveraging Electronic Tablets (CHICLET)}

CHICLET is an electronic tablet based interface for the CHICA system. It is initially developed to screen patients or their caregivers in the waiting room and to capture vital signs information from the nursing staff. Technically, to achieve CHICLET based functionality CHICA's clinical decision 
support (CDS) engine is exposed via web services. CHICA web services use standard HTTPS protocol for communication between the CHICA server and the CHICLET client (android OS application) and this communication is secured with multiple layers of authentication. ( Figures 2 a \& b) No patient data is ever stored on CHICLET device. Instead the PSF questions and responses reside in the device session memory which is erased at the end of the session. Other security precautions are also implemented for CHICLET deployment, for example Meraki software (www.cisco. com) tracks and monitors all electronic tablets deployed. This software allows for remote messaging, remote locking, and remote wiping of a device if one becomes lost or stolen. Simple Object Access Protocol (SOAP) is used to exchange patient information between CHICA and CHICLET in realtime and this exchange allows for algorithmic execution of computational guidelines in CHICA's clinical decision support engine using most recent patient information, i.e. often from patient's screen in the waiting room. Using CHICLET, many enhancements to the CDSS can be based on the real-time exchange of patient information, for example to ask more detailed screening questions (based on previous responses) of patients. However, since the aim of this pilot study is to evaluate CHICLET compared to the existing scan-able paper interface, we did not implement these enhancements.

\section{CHICLET based intervention}

For the purposes of this study, CHICLET based PSF presents the same screening questionnaire as the scan-able paper based PSF. The maximum questions asked on PSF remain the same (i.e. 20) on both scan-able paper and CHICLET PSF versions. Both PSF versions implement questionnaire in English and Spanish and a choice to answer in either. However, due to the limitations of the device screen size for readability, CHICLET based PSF displays four pages with maximum of five questions on each page compared to all questions on the paper version of the PSF. For this pilot study, two electronic tablet devices (Samsung Nexus 10 tablets with android OS) were deployed with CHICLET interface in one of the smaller pediatric clinics (consisting of three medical doctors, two medical assistants and one full time nurse) using the CHICA system. This clinic (henceforth intervention clinic) was chosen based on workflow constraints, and staff's desire to try new technology. Due to the limited number of available devices for use, we limited this pilot study to children ages zero to twelve years, however, children with siblings at the visit and those receiving M-CHAT were excluded. However, this age group constituted the most frequent age at which children visit our clinics for care.

\section{Caregiver Survey}

We surveyed parents and caregivers to assess the usability of electronic tablets (CHICLET) in a clinical setting. The caregiver survey consists of twelve questions for measuring usability on five point Likert scale and was offered in both English and Spanish. The survey also asked about caregiver's relationship to the child, number of children in the household and caregiver suggestions or comments about using the electronic tablet device (Supplementary Appendix 1) Parents or caregivers (> eighteen years of age) with children less than or up to twelve years of age were randomly invited to complete an anonymous survey about their experience with CHICLET. This survey was offered either at the end of their CHICLET session or at the end of the clinic visit depending upon workflow demands. The survey was administered on paper by an experienced Research Assistant (RA) from our practice-based research network - Pediatric Research Network or PResNet. It facilitates pediatric patient recruitment and surveys in our outpatient settings and supports a broad range of research initiatives. PResNet RAs have been well trained in working with human subjects, following protocols and implementing accurate data collection methods. Participants from a family unit were asked to complete the survey only once during the entire study. A \$5 gift card was offered to those who participated and the anonymous survey took no more than 5 tol0 minutes to administer by experienced RAs. 


\section{Staff Survey}

Medical assistants and nursing staff who used CHICA on a regular basis, for example to record patient's vital sign information or to room the patient, were asked to complete a brief survey with questions about perceived usability of CHICLET and its impact on workflow. The survey consisted of six questions of usability on five point Likert scale and asked the staff members about their number of years of job experience using CHICA as well as any suggestions or comments that they may have about the use of CHICLET. (Supplementary Appendix 2) The staff members provided written informed consent before survey administration and also completed the survey only once (at different times) during the entire study. At the end of the study, participating staff were compensated with $\$ 10$ gift card.

Surveys for this study were conducted between March 2013 and June 2013.

\section{Analysis}

The following de-identified data items were extracted from CHICA's database for this study for the study population, i.e. children zero to twelve years of age: number of questions asked on scan-able or CHICLET based PSF for the particular visit, number of questions answered for that visit, time point in the study (pre-intervention or intervention period) and socio-demographics (race, gender, insurance). The following analyses were performed using Stata software version 12 (StataCorp, College Station, TX, www.stata.com). Results were considered statistically significant at $\mathrm{p}<0.05$.

1. To determine if data collected through CHICLET were as complete as data collected using the scan-able paper based PSF data were compared for children who visited the intervention clinic before and after the implementation of CHICLET. We measured scan-able paper based PSF and CHICLET based PSF completion rates. Completion rate was defined as the proportion of questions answered (of the total number of questions asked) on a given screening questionnaire. Data completion rates for two months pre-intervention (January, February 2013), and two months during intervention (March, April 2013) were compared using chi-square test. Data from a comparable clinic (consisting of three medical doctors, one nurse practitioner, three medical assistants and one full time nurse) are used to compare PSF completion rates in pre, intervention and post intervention (May, June 2013) periods.

2. We evaluated the level of responses on the caregiver survey to measure caregiver CHICLET usability

3. We evaluated the level of responses on staff survey to measure staff CHICLET usability.

Indiana University Institutional Review Board approved this study (IRB \# 1301010447).

\section{Results}

Data for 853 children between zero and twelve years of age was available from CHICA's database for this study. These children visited the intervention clinic at least once during the study period. Almost an equal number of children visited during the pre-intervention period $(n=441,52 \%)$ as during the intervention period $(n=412,48 \%)$. There were no significant differences for race, gender or insurance category $\left(\mathrm{X}^{2}=6.7, \mathrm{p}=0.150\right)$ between the pre-intervention and intervention periods. Please see $>$ Table 1 for socio-demographic characteristics of the study population by both periods. At these visits, CHICA asked parents or caregivers up to twenty screening questions using either scan-able paper or CHICLET based PSF (based on the expected value of information as described in the methods section above). Of these parents or caregivers, 708 (83\%) were asked twenty questions, $109(13 \%)$ were asked between eleven and nineteen questions and $36(<5 \%)$ were asked ten or fewer questions. 


\section{PSF/CHICLET Completion rates}

Overall parents or caregivers answered one or more screening questions on 528 (62\%) PSFs, of which 229 (43\%) PSFs had one or more questions answered during the pre-intervention period using scan-able paper and 299 (57\%) PSFs had one or more questions answered during the intervention period using CHICLET. During the study period, CHICA generated 1501 scan-able paper or CHICLET based PSFs for the intervention clinic for all pediatric age groups. In general, parents or caregivers completed fewer questions on scan-able PSF when compared to CHICLET based PSF. For example, in the pre-intervention period, $50 \%$ of scan-able PSFs were partially completed in the intervention clinic while in a comparable clinic only $47 \%$ of PSFs were partially completed during this time period. This is in contrast to more than $70 \%$ of CHICLET based PSFs fully completed in the intervention clinic during the intervention period showing a significant increase in the number of overall questions answered. Please see $>$ Figure 3 for details. The proportion of parents and caregivers who answered the PSF completely (i.e. 100\% completion rate) was higher in the intervention period ( $n=234,78 \%$ ) when compared to the pre-intervention period $(n=138,60 \%)$. The $18 \%$ difference in completion rate corresponds to a $30 \%$ relative increase in the intervention period and is statistically significant $\left(\mathrm{X}^{2}=32, \mathrm{p}=0.002\right)$.

\section{Caregiver CHICLET Usability}

Parents and caregivers of the 412 children who visited the intervention clinic during the intervention period were eligible to receive a caregiver survey. Of these, 113 (27\%) parents or caregivers (97 mothers, 8 fathers, 6 others and 2 not known) accepted the invitation to complete the caregiver survey at the time of their visit. Their survey data are analyzed for usability of CHICLET interface and presented in this study. Overall caregivers responded positively for answering screening questions using CHICLET. The majority of caregivers reported that it was easy to use CHICLET - to hold (96\%), follow instructions (98\%), answer questions (95\%) and navigate from page to page (95\%). Seventy two percent also reported it was easy to turn on the CHICLET device (if it was turned off). Only a very small percentage of caregiver respondents reported that CHICLET interface was hard to read $(<1 \%)$, made errors $(1 \%)$ and that a lot of questions were asked $(3 \%)$ or they did not know how to answer questions (3\%) or got tired of answering the same questions on it $(4 \%)$.

When asked if the caregivers liked to answer questions on the paper PSF, $44 \%$ disagreed (13\% somewhat disagreed) that they liked answering questions on the paper PSF. Forty seven percent remained neutral, and $8 \%$ agreed (4\% strongly agreed). When asked if answering questions on CHICLET made the caregivers think of things to talk to their doctor, the majority agreed (65\% agreed $40 \%$ strongly agreed, and $25 \%$ somewhat agreed).

\section{Staff CHICLET Usability}

Due to limited number of staff available in the intervention clinic, only three staff surveys were done during the study period. All three staff survey respondents strongly agreed about the usability of CHICLET for entering patient's vital sign information using CHICLET based PSF. They agreed that CHICLET based input worked error free and it made their jobs easier in the clinical setting. All three staff also strongly agreed that they did not like scanning paper forms and two strongly disagreed that they like manual verification of scanned forms. Only one staff member somewhat disagreed regarding the verification of scanned forms. The staff also commented on how they should use CHICLET for all patient ages and not only for children zero to twelve years of age (as used for this study). Among the three staff surveyed, two had five to seven years of job experience and all three had been using CHICA between two and four years. Contrary to staff's concerns, there were no electronic tablets lost or stolen in this pilot study. 


\section{Discussion}

We have successfully piloted an electronic tablet based interface, CHICLET, with our pediatric clinical decision support system, CHICA, and evaluated its usability to capture patient's data in pediatric waiting rooms. Furthermore, we have demonstrated that a patient-facing electronic tablet based interface can substantially improve completion rates for patient reported data without interfering with existing clinical workflow. Our results are comparable to past research using electronic tablet devices in children and adolescent studies, for example to boost survey response rates or to understand gender differences for comprehensive health risk assessment [31, 32]. However, these studies did not address the use of electronic tablet devices in routine pediatric care. As CHICLET successfully replaced the scan-able paper based PSF in our setting, it effectively supported the underlying screening processes for driving clinical decision support implemented in CHICA [25-30, 33, 34]. Because CHICLET is designed to replace the scan-able paper interface with an electronic version, its use has implications for clinical decision support enhancements as well as opportunities for research in the area of patient engagement. For example, the percent of fully completed CHICLET based screenings in our study is much higher when compared to the scan-able paper based PSF. This improvement suggests that CHICLET may be able to implement "drill-down" screening processes to optimize sensitivity and specificity of screening while engaging patients, for example, to ask questions pertinent to their own care of their physicians [35-40]. Of the limited number of caregivers surveyed, majority agreed that CHICLET based screening prior to their physician encounter was helpful in reminding them of things to talk to their doctor about during the encounter. Similarly, the staff agreed that CHICLET use helped them in error-free collection of patient's vital sign information.

Based on this study, we believe that electronic tablet based interface to a CDSS presents many advantages for data capture in clinical settings. We believe, the completion rate in our study is much higher with electronic tablets when compared to the paper based screening because the caregivers are presented a set number of questions per page (on CHICLET) as opposed to the entire questionnaire (on a scan-able PSF). Such presentation perhaps helps in minimizing the selection bias, the caregiver's tendency to selectively answer questions, though they can selectively answer questions using either medium. Similarly, recording the vital signs information using electronic tablets helps nursing staff minimize errors because they only handle it once at the point of care as opposed to transcribing from or verifying the paper version. In the long term, an electronic tablet based interface may also save time for the user, time that is needed to manually verify mis-scanned forms in a scan-able paper based interface. However, it needs to be studied if this efficiency is at the cost of deploying and securing electronic tablets in a clinical setting.

As with all such studies, our study has some limitations. Due to a fixed number of electronic tablet devices that were available for use, we limited this pilot study to children up to12 years of age. Another limitation of our study is that the intervention clinic had an existing well-defined workflow (as with all the clinics implementing the CHICA system) and we only replaced the screening form with an electronic tablet version. A detailed workflow assessment may be needed before electronic tablet interfaces can be deployed in other settings. We also did not study other potentially important measures for deployment such as time on task for electronic tablet verses paper based system, or computer or educational literacy required for the intervention. In this study, as we focused on screening processes and patient-facing side of the technology, we did not survey the providers. We also have a limited number of surveys for assessing staff's usability of CHICLET. Therefore, further studies are needed to assess both staff and provider acceptance of the technology in larger clinics. Though we measured caregivers usability from a large sample of the clinic population it is feasible that those who agreed to participate may have self-selected. Finally, the staff may have boosted the completion rates in this study, by providing help to caregivers at times (for e.g. in an exam room while the caregiver changed baby's diaper). However, on the same token, our completion rates reflect the staff's willingness to help and try new technology. Regardless, parents of young children (as in our study) are young themselves, and therefore, are more receptive to technology. Therefore, when deploying electronic tablet technology in older populations and in different healthcare settings, there may be additional costs to consider, for example, cost of training, and securing wireless networks. 
Further studies are needed to evaluate usefulness in other care settings as well as study future use for patient engagement and satisfaction.

\section{Conclusion}

Electronic tablet devices are a viable interface for use in pediatric waiting rooms to capture patient reported data. In future, these devices can not only be used for screening but also for capturing patient self-report of preferences, risks, outcomes and barriers, particularly in many pediatric chronic conditions and in populations speaking varied languages. Such use may perhaps result in greater patient engagement and satisfaction however this needs to be studied formally. Therefore, many future interventions focused on engaging patients in their own care can be designed using electronic tablet devices in a clinical setting.

\section{Clinical Relevance Statement}

An existing scan-able paper based interface to a long running and widely studied pediatric CDSS has been limiting interactive and iterative assessment of patient self-report in pediatric waiting rooms. In this study, we replace the existing scan-able paper based interface with an electronic tablet based version and evaluate usability via caregiver and staff surveys and changes in patient question completion rates as a result. The patient completion rates for electronic version are significantly different from scan-able paper version and the caregivers and staff are supportive for the use of electronic tablets in routine pediatric care, however, to evaluate the use of such devices for advanced clinical decision support and studying patient engagement, further studies are needed.

\section{Conflict of Interest}

The authors declare that they have no conflict of interest in the research.

\section{Human Subjects Protections}

The study was performed in compliance with the World Medical Association Declaration of Helsinki on Ethical Principles for Medical Research Involving Human Subjects, and was reviewed by the Indiana University Institutional Review Board.

\section{Acknowledgements}

The authors would like to thank Eskenazi health, PResNET and Child Health Informatics Research and Development Lab (CHIRDL) for this study. The views expressed in this article are those of the authors and do not necessarily represent those of Indiana University. 


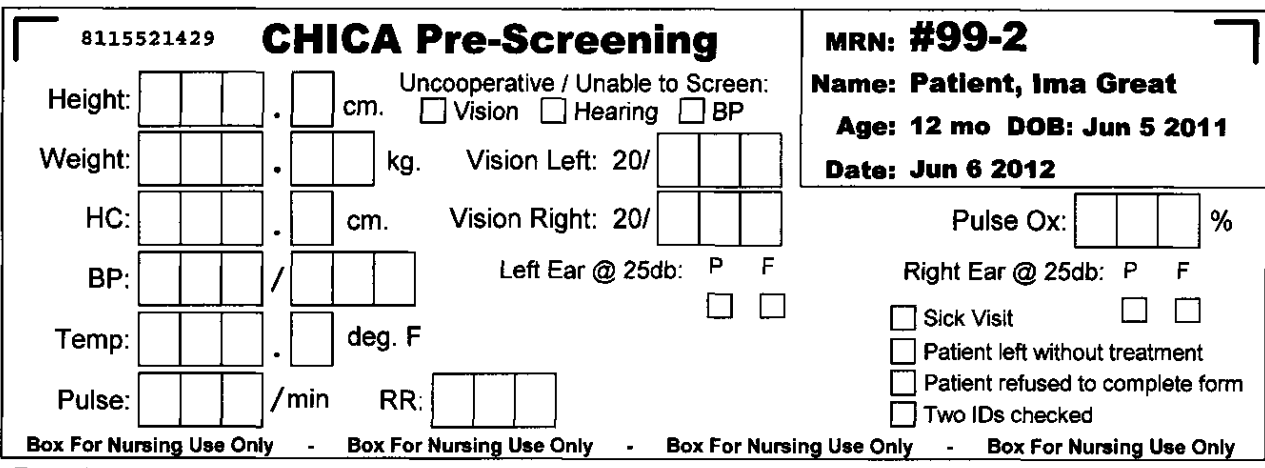

Parents: Thank you for answering these questions about your child. The answers will help your doctor provide better quality of care. If your child is age 11 or older, he/she should answer the questions privately. Answers are confidential, but if you prefer not to answer that is allowed. You may want to talk about these questions with your doctor.

Please fill in the circles completely with a pencil or pen.
(1) (1) Is Ima allergic to any medicines?
() () Is Ima having pain today?

$\mathbf{Y} \mathbf{N}$

(1) (1) Can Ima play pat-a-cake with someone without any help, such as helping her clap her hands?

(1) (1) Does Ima repeat the same sounds several times in a

(1) Do you feel safe in your home?

(1) (D) Has your partner kicked, hit, or slapped you?

(-) In the past 7 days, have you felt scared or panicky for not a very good reason?

(1) In the past 7 days, have you blamed yourself unnecessarily when things went wrong?

(1) In the past 7 days, have you been anxious or worried for no good reason?

(1) Have you set your hot water heater to less than 120 degrees $F$ or 49 degrees $C$ ?

() () Does Ima watch some TV most days?

(1) Have you attached the Poison Center phone number to the phone in Ima's home?

(1) Do you know what to do if ima is choking?

(1) (1) Has Ima ever been alone in or near a bathtub, swimming or wading pool, or other body of water?

(1) (1) Has your partner or another adult threatened or hurt your children?

(1) Does ima ever use a bottle?

(1) Has Ima seen a dentist this year?

(-) (1) Does Ima sleep with a bottle or breastfeed all night?

(1) () Was Ima's birth weight less than five pounds and eight ounces ( 2500 grams)?

(1) Is Ima on the WIC program?
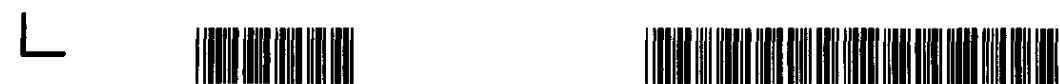

Fig. 1 Scan-able paper based PSF 


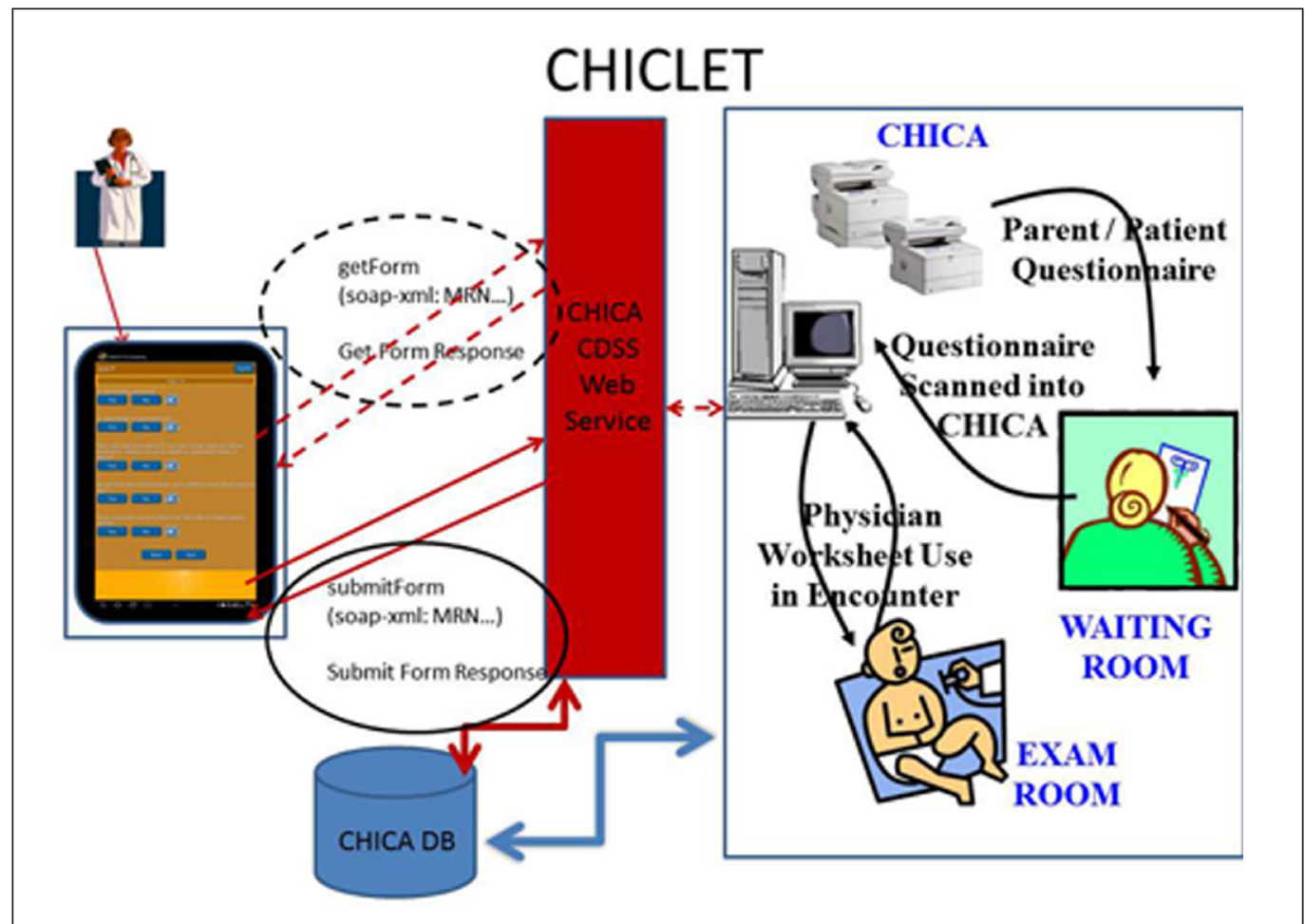

Fig. 2a CHICA Web Services

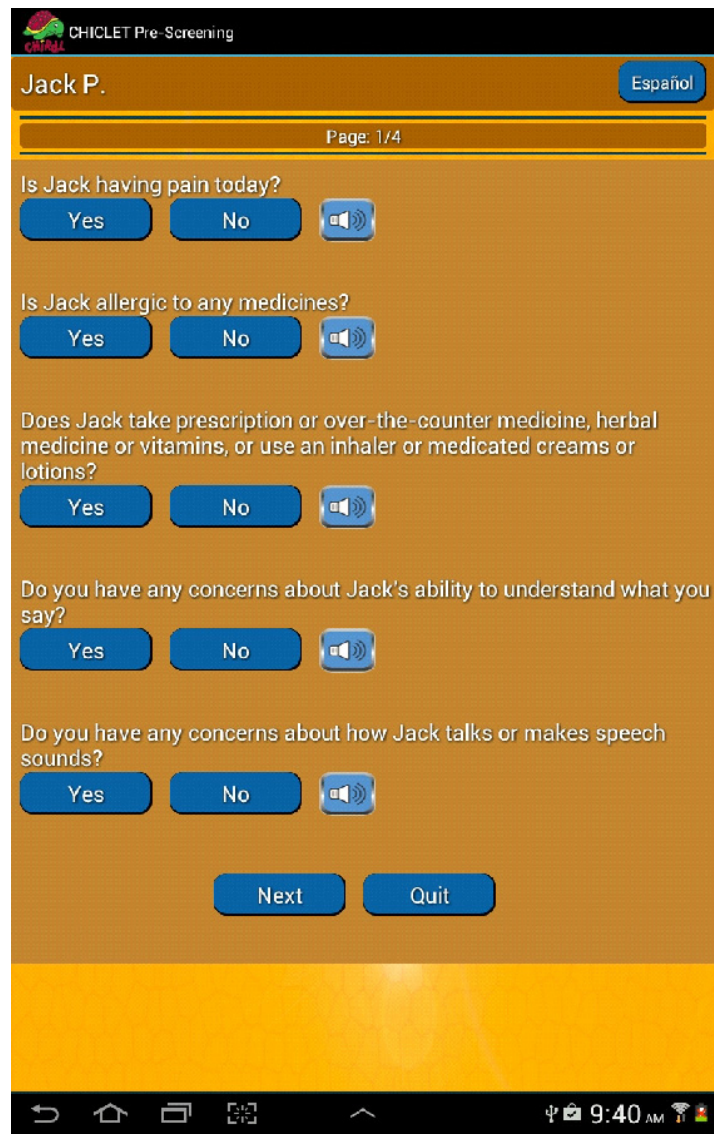

Fig. 2b CHICLET Interface 


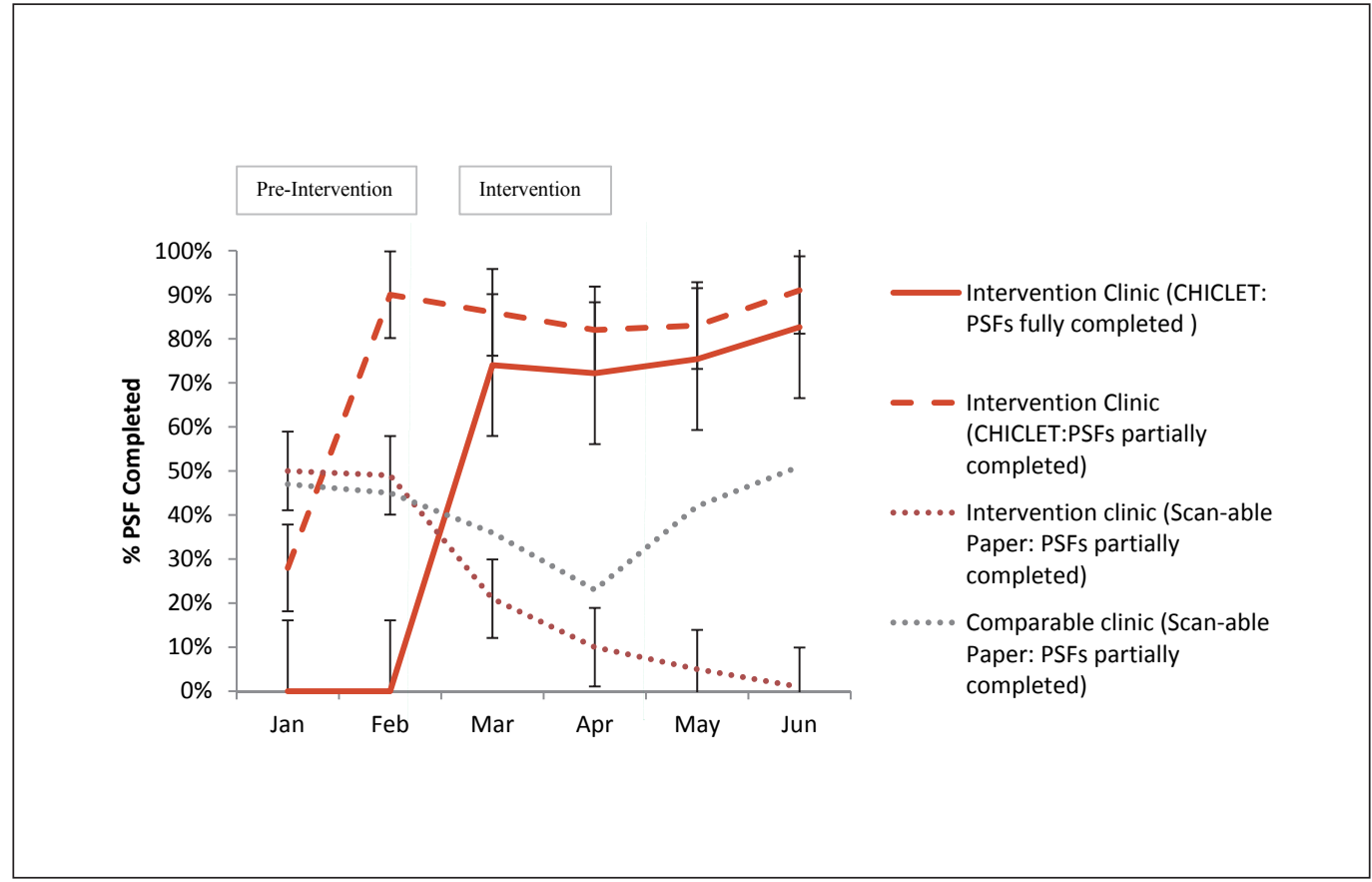

Fig. 3 PSF Completion Rate (Intervention clinic: 3 Medical doctors, 2 Medical Assistants, 1 nurse, patient volume: 20 - 45 per day. Comparable clinic: 3 Medical doctors,3 Medical Assistants, 1 nurse practitioner, 1 nurse, patient volume: $20-60$ per day) 
Table 1 Demographics of study population (children ages 0 to 12 years) $(n=853)$

\begin{tabular}{|l|r|r|r|}
\hline & Intervention & Pre-Intervention & Total (\%) \\
\hline Race/Ethnicity & & & \\
\hline Black & 342 & 344 & $686(81.1 \%)$ \\
\hline Latino & 42 & 53 & $95(11.2 \%)$ \\
\hline White & 20 & 35 & $55(6.5 \%)$ \\
\hline Other / Unknown & 0 & 1 & $1(<\%)$ \\
\hline Asian Pacific / Islander & 3 & 6 & $9(1.1 \%)$ \\
\hline Sex & 182 & & $378(44.3 \%)$ \\
\hline Female & & 196 & $19(2.3 \%)$ \\
\hline Insurance Category & 8 & 11 & $33(3.9 \%)$ \\
\hline No Insurance & 15 & 18 & $787(93.8 \%)$ \\
\hline Private & 376 & 411 & \\
\hline Public & & & \\
\hline
\end{tabular}




\section{References}

1. Hing E, Hsiao CJ. Electronic medical record use by office-based physicians and their practices: United States, 2007. Natl Health Stat Report 2010(23): 1-11. Epub 2010/07/17. PubMed PMID: 20632518.

2. Kreps GL, Neuhauser L. New directions in eHealth communication: opportunities and challenges. Patient Educ Couns 2010; 78(3): 329-336. Epub 2010/03/06. doi: S0738-3991(10)00022-4 [pii] 10.1016/j.pec.2010.01.013. PubMed PMID: 20202779.

3. Garner AS, Shonkoff JP, Siegel BS, Dobbins MI, Earls MF, McGuinn L,Pascoe J, Wood DL. Early childhood adversity, toxic stress, and the role of the pediatrician: translating developmental science into lifelong health. Pediatrics 2012; 129(1): e224-e231. Epub 2011/12/28. doi: peds.2011-2662 [pii] 10.1542/peds.2011-2662. PubMed PMID: 22201148.

4. Mangione-Smith R, DeCristofaro AH, Setodji CM, Keesey J, Klein DJ, Adams JL, Schuster MA, McGlynn EA. The quality of ambulatory care delivered to children in the United States. N Engl J Med 2007; 357(15): 1515-1523. Epub 2007/10/12. doi: 357/15/1515 [pii] 10.1056/NEJMsa064637. PubMed PMID: 17928599.

5. Koppel R, Kreda DA. Healthcare IT usability and suitability for clinical needs: challenges of design, workflow, and contractual relations. Stud Health Technol Inform 2010; 157: 7-14. Epub 2010/06/15. PubMed PMID: 20543360.

6. Anand V, Biondich PG, Liu G, Rosenman M, Downs SM. Child Health Improvement through Computer Automation: the CHICA system. Stud Health Technol Inform 2004; 107(Pt 1): 187-191. Epub 2004/09/14. doi: D040004973 [pii]. PubMed PMID: 15360800.

7. Biondich PG, Anand V, Downs SM, McDonald CJ. Using adaptive turnaround documents to electronically acquire structured data in clinical settings. AMIA Annu Symp Proc 2003: 86-90. Epub 2004/01/20. doi: D030003628 [pii]. PubMed PMID: 14728139; PubMed Central PMCID: PMC1480055.

8. Biondich PG, Downs SM, Anand V, Carroll AE. Automating the recognition and prioritization of needed preventive services: early results from the CHICA system. AMIA Annu Symp Proc 2005: 51-55. Epub 2006/06/17. doi: 58031 [pii]. PubMed PMID: 16779000; PubMed Central PMCID: PMC1560868.

9. Downs SM, Anand V, Dugan TM, Carroll AE. You can lead a horse to water: physicians' responses to clinical reminders. AMIA Annu Symp Proc 2010; 2010: 167-171. Epub 2011/02/25. PubMed PMID: 21346962; PubMed Central PMCID: PMC3041342.

10. Downs SM, Carroll AE, Anand V, Biondich PG. Human and system errors, using adaptive turnaround documents to capture data in a busy practice. AMIA Annu Symp Proc 2005: 211-215. Epub 2006/06/17. PubMed PMID: 16779032; PubMed Central PMCID: PMC1560802.

11. Anand V, Biondich PG, Liu G, Rosenman M, Downs SM. Child Health Improvement through Computer Automation: the CHICA system. Stud Health Technol Inform 2004; 107(Pt 1): 187-191. Epub 2004/09/14. PubMed PMID: 15360800 .

12. Biondich PG, Downs SM, Anand V, Carroll AE. Automating the recognition and prioritization of needed preventive services: early results from the CHICA system. AMIA 2005; Annual Symposium Proceedings/ AMIA Symposium 51-55. PubMed PMID: 16779000.

13. Downs SM, Biondich PG, Anand V, Zore M, Carroll AE. Using Arden Syntax and adaptive turnaround documents to evaluate clinical guidelines. AMIA Annu Symp Proc 2006: 214-218. Epub 2007/01/24. PubMed PMID: 17238334; PubMed Central PMCID: PMC1839583.

14. Biondich PG, Anand V, Downs SM, McDonald CJ. Using adaptive turnaround documents to electronically acquire structured data in clinical settings. AMIA Annu Symp Proc 2003: 86-90. Epub 2004/01/20. PubMed PMID: 14728139; PubMed Central PMCID: PMC1480055.

15. Downs SM, Carroll AE, Anand V, Biondich PG. Human and system errors, using adaptive turnaround documents to capture data in a busy practice. AMIA 2005: 211-215. PubMed PMID: 16779032.

16. Mamlin BW, Biondich PG, Wolfe BA, Fraser H, Jazayeri D, Allen C, Miranda J, Tierney WM. Cooking up an open source EMR for developing countries: OpenMRS - a recipe for successful collaboration. AMIA 2006: 529-533. Epub 2007/01/24. doi: 86578 [pii]. PubMed PMID: 17238397; PubMed Central PMCID: PMC1839638.

17. McDonald CJ, Overhage JM, Tierney WM, Dexter PR, Martin DK, Suico JG,Zafar A, Schadow G, Blevins L, Glazener T, Meeks-Johnson J, Lemmon L, Warvel J, Porterfield B, Warvel J, Cassidy P, Lindbergh D, Belsito A, Tucker M, Williams B, Wodniak C. The Regenstrief Medical Record System: a quarter century experience. International journal of medical informatics 1999; 54(3): 225-253. PubMed PMID: 10405881.

18. Anand V, Biondich PG, Carroll AE, Downs SM. Tailoring Interface for Spanish Language: A Case Study with CHICA System. Proceedings of the 1st International Conference on Human Centered Design: Held as Part of HCI International 2009; San Diego, CA. 1601681: Springer-Verlag; 2009; 398-407.

19. Hripcsak G, Ludemann P, Pryor T, Wigertz O, Clayton P. Rationale for the Arden Syntax. Computers and Biomedical Research 1994; 4: 291-324. 
20. Jenders RA, Hripcsak G, Sideli RV, DuMouchel W, Zhang H, Cimino JJ, Johnson SB, Sherman EH, Clayton PD. Medical decision support: experience with implementing the Arden Syntax at the Columbia-Presbyterian Medical Center. Proc Annu Symp Comput Appl Med Care 1995: 169-173. PubMed PMID: 8563259 .

21.Downs SM, Uner H. Expected value prioritization of prompts and reminders. Proceedings / AMIA Annual Symposium AMIA Symposium 2002: 215-219. Epub 2002/12/05. doi: D020002217 [pii]. PubMed PMID: 12463818.

22. Squires J, Bricker D, Potter L. Revision of a parent-completed development screening tool: Ages and Stages Questionnaires. J Pediatr Psychol 1997; 22(3): 313-328. Epub 1997/06/01. PubMed PMID: 9212550.

23. Wolraich ML, Lambert W, Doffing MA, Bickman L, Simmons T, Worley K. Psychometric properties of the Vanderbilt ADHD diagnostic parent rating scale in a referred population. J Pediatr Psychol 2003; 28(8): 559-567. Epub 2003/11/07. PubMed PMID: 14602846.

24. Diana L. Robins PD. Modified Checklist for Autism in Toddlers (M-CHAT) 1999.

25. Anand V, Carroll AE, Downs SM. Automated primary care screening in pediatric waiting rooms. Pediatrics 2012; 129(5): e1275-e1281. Epub 2012/04/18. doi: 10.1542/peds.2011-2875. PubMed PMID: 22508925; PubMed Central PMCID: PMC3340595.

26. Carroll A, Anand V, Dugan T, Sheley M, Xu S, SM D. Increased Physician Diagnosis of Asthma with the CHICA Decision Support System. Pediatric Allergy, Immunology, and Pulmonology 2012; 25(3): $168-171$.

27. Carroll AE, Bauer NS, Dugan TM, Anand V, Saha C, Downs SM. Use of a computerized decision aid for ADHD diagnosis: a randomized controlled trial. Pediatrics 2013; 132(3): e623-e629. Epub 2013/08/21. doi: 10.1542/peds.2013-0933. PubMed PMID: 23958768; PubMed Central PMCID: PMC3876764.

28. Carroll AE, Bauer NS, Dugan TM, Anand V, Saha C, Downs SM. Use of a Computerized Decision Aid for Developmental Surveillance and Screening: A Randomized Clinical Trial. JAMA pediatrics 2014. Epub 2014/07/16. doi: 10.1001/jamapediatrics.2014.464. PubMed PMID: 25022724.

29. Carroll AE, Biondich P, Anand V, Dugan TM, Downs SM. A randomized controlled trial of screening for maternal depression with a clinical decision support system. J Am Med Inform Assoc 2013; 20(2): 311-316. Epub 2012/06/30. doi: 10.1136/amiajnl-2011-000682. PubMed PMID: 22744960; PubMed Central PMCID: PMC3638168.

30. Carroll AE, Biondich PG, Anand V, Dugan TM, Sheley ME, Xu SZ, Downs SM. Targeted screening for pediatric conditions with the CHICA system. J Am Med Inform Assoc 2011; 18(4): 485-490. Epub 2011/06/16. doi: 10.1136/amiajnl-2011-000088. PubMed PMID: 21672910; PubMed Central PMCID: PMC3128399.

31. Kadivar H, Thompson L, Wegman M, Chisholm T, Khan M, Eddleton K, Muszynski M, Shenkman E. Adolescent views on comprehensive health risk assessment and counseling: assessing gender differences. The Journal of adolescent health : official publication of the Society for Adolescent Medicine 2014; 55(1): 24-32. Epub 2014/03/13. doi: 10.1016/j.jadohealth.2013.12.002. PubMed PMID: 24613096.

32. Parker MJ, Manan A, Urbanski S. Prospective evaluation of direct approach with a tablet device as a strategy to enhance survey study participant response rate. BMC research notes 2012; 5: 605. Epub 2012/11/01. doi: 10.1186/1756-0500-5-605. PubMed PMID: 23110849; PubMed Central PMCID: PMC3522021.

33. Bauer NS, Anand V, Carroll AE, Downs SM. Secondhand Smoke Exposure, Parental Depressive Symptoms and Preschool Behavioral Outcomes. Journal of pediatric nursing 2014. Epub 2014/07/16. doi: 10.1016/j.pedn.2014.06.004. PubMed PMID: 25017291.

34.Downs SM, Zhu V, Anand V, Biondich PG, Carroll AE. The CHICA smoking cessation system. AMIA Annu Symp Proc 2008: 166-170. Epub 2008/11/13. PubMed PMID: 18998823; PubMed Central PMCID: PMC2655976.

35. Brennan PF, Strombom I. Improving health care by understanding patient preferences: the role of computer technology. Journal of the American Medical Informatics Association: JAMIA 1998; 5(3): 257-262. Epub 1998/06/03. PubMed PMID: 9609495; PubMed Central PMCID: PMC61299.

36. Eysenbach G. Consumer health informatics. Bmj 2000; 320(7251): 1713-1716. Epub 2000/06/23. PubMed PMID: 10864552; PubMed Central PMCID: PMC1127483.

37. Jimison HB, Sher PP, Jimison JJB. Decision Support for Patients Clinical Decision Support Systems. In: Berner ES, editor.: Springer New York; 2007; 249-261.

38. Ruland CM. Handheld technology to improve patient care: evaluating a support system for preferencebased care planning at the bedside. Journal of the American Medical Informatics Association: JAMIA 2002; 9(2): 192-201. Epub 2002/02/28. PubMed PMID: 11861634; PubMed Central PMCID: PMC344576.

39. Ruland CM, Bakken S. Developing, implementing, and evaluating decision support systems for shared decision making in patient care: a conceptual model and case illustration. J Biomed Inform 2002; 35(5-6): 313-321. Epub 2003/09/13. PubMed PMID: 12968780. 
40.van der Weijden T, Legare F, Bolvin A, Burgers JS, van Veenendaal H, Stiggelbout AM, Faber M, Elwyn G. How to integrate individual patient values and preferences in clinical practice guidelines? A research protocol. Implementation science: IS 2010; 5: 10. 